\title{
Clostridium Sordellii Colitis
}

National Cancer Institute

\section{Source}

National Cancer Institute. Clostridium Sordellii Colitis. NCI Thesaurus. Code C35634.

Inflammation of the colon resulting from Clostridium Sordelli. 